\title{
Editorial
}

\section{Special issue on business process lifecycle: design, deployment, operation and evaluation}

This special issue on Business Process Lifecycle: Design, Deployment, Operation and Evaluation follows the 9th Workshop on Business Process Modeling, Development and Support (BPMDS'08) organized in conjunction with CAiSE'08, which was held in Montpellier, France in June 2008. The special issue is targeted at both researchers and practitioners in the information systems (in the broad sense) community. It is the result of an open call for papers in which both papers from BPMDS'08 and contributions from the business process engineering community were selected following several blind review rounds. We present extensively modified versions of five BPMDS'08 papers and two new contributions.

In this special issue, we used the business process lifecycle model shown in Figure 1. It comprises three phases: design, deployment, operation and evaluation. The design phase begins with the requirements definition and ends with the implementation of the business process. In the deployment phase, the business process is tested for fulfilling the customer's requirements. After successful testing, the business process is released to operation. In the operation and evaluation phase, the business process is measured and analyzed to identify problems and potential improvements. Thus, the continuous improvement requirement is the force that 'drives' the wheel of the business process lifecycle.

During the BPMDS workshops prior to BPMDS'08, various issues that could be related to different but isolated phases in the lifecycle of a business process have been discussed. In the BMPDS08 workshop, the focus was set on the interaction between two or more phases of the lifecycle, as well as the holistic view of the whole lifecycle. Furthermore, the relationships between the phases have been investigated. The papers in this special issue remain faithful to this focus.

\section{DESIGN TO DEPLOYMENT}

The paper by Adam et al., 'From business processes to software services and vice versa - an improved transition through service-oriented requirements engineering', draws our attention on the fact that flexibility and efficiency are strongly dependent on the information technology (IT) used; and IT and information systems are often considered as an enabler for the business success. Nevertheless, whereas process design and evaluation are performed by business people (supported by IT), implementation and execution are pure IT-world tasks. The so-called business-IT gap leads to costly and time-consuming human intervention when business process models are to be brought into production or vice versa. Because the transition from the design to the deployment phase is a central one with regard to enterprise flexibility, the activity of business process automation aims at lowering the effort for developing and releasing information systems from business process models by automating this transformation. Since the last decade, the paradigm of service-oriented architecture (SOA) is considered as a partner for business process management (BPM), providing mechanisms for making information systems and thus enterprises more agile and flexible. The basic idea of SOA is the restructuring of IT landscapes into loosely coupled, independent software services. These software services, which can be recursively integrated into more coarse-grained services, should allow the reuse of existing functionality in order to shorten the time between process design and system implementation when business requirements change. The main challenge in developing serviceoriented information systems is therefore an insufficient methodology for the refinement and mapping of business processes to existing service infrastructures. 


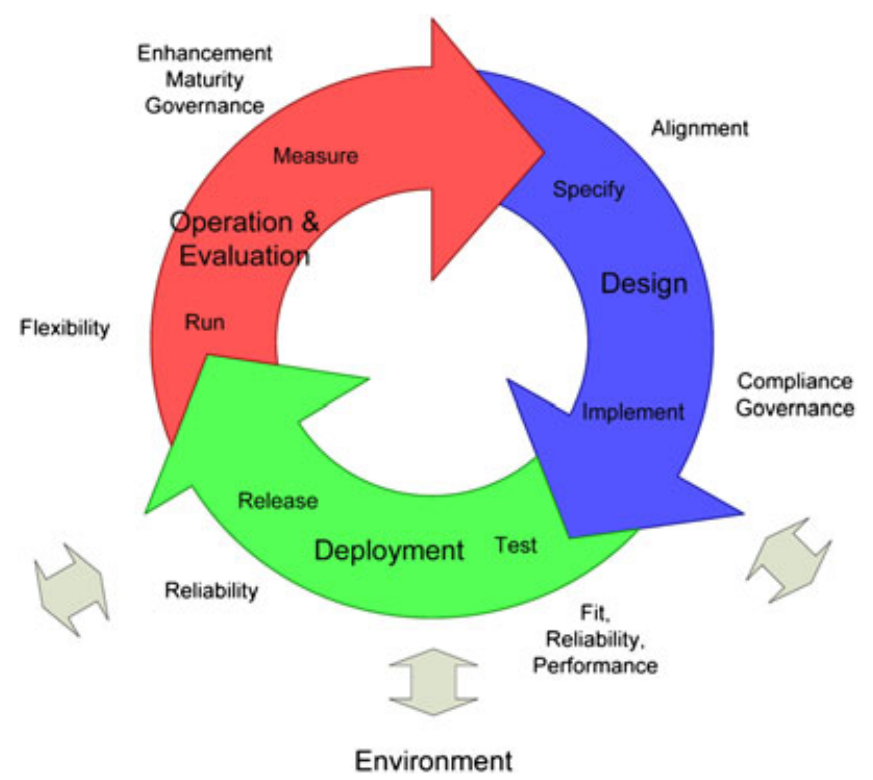

Figure 1. Business process lifecycle phase and their purposes.

This paper extends the previous work of the authors on the integration of BPM and SOA by providing more details on the methods developed, by integrating them in a common framework and by validating them in two case studies. The service-oriented requirements engineering is proposed as a discipline aiming at aligning BPM and SOA more explicitly. Furthermore, a method for developing reusable software services based on a representative set of business processes to be supported is introduced. An approach for service-aware business process design is also introduced.

The paper by Ukor et al., 'Service selection and horizontal multi-sourcing in process-oriented capability outsourcing', addresses a new dimension introduced by the SOA in process design and development. Authors recall us that multiple service providers often exist for a required business capability. Accordingly, service requesters must select an optimal set of providers from discovered service providers based on a combination of selection criteria through a process known as service selection. Traditionally, the use of business services in a process involves determining all possible execution paths and their corresponding services during the design phase. When circumstances change during the operation phase and a new service must be used in some part of the process, the business process is redesigned and transitioned into the operation phase all over again. This traditional approach to service-oriented capability outsourcing is unable to cope with new challenges related to dynamic service discovery and delivery such as the Cloud as it is not always possible to know which services would be available at the time process instances are enacted. To address this problem, a dynamic approach to service selection and integration, which incorporates a mini-lifecycle within the context of process instances is proposed by the authors.

This paper addresses a new problem model for the service selection optimisation problem with support for horizontal multi-sourcing, and evaluates three solution approaches to the problem. The model provides support for horizontal multi-sourcing through a concept called request token, which characterises the different horizontal partitions of a business capability. Request tokens are used by service providers as the basic unit for describing their service offerings as well as by service requesters for selecting an optimal set of providers for a given process instance. Four controlled experiments were performed for evaluation.

\section{DESIGN TO EVALUATION TO (RE)DESIGN}

The paper by Zugal et al., 'Toward enhanced lifecycle support for declarative processes', addresses a critical success factor in applying process-aware information systems (PAISs), namely the possibility 
of flexibly dealing with process changes. The referenced literature shows that to handle the need for flexible PAISs, competing paradigms enabling process changes and process flexibility have been developed during the last decade; one of them is the declarative process modelling. All these approaches relax the strict separation of build-time (i.e., design) and run-time (i.e., operation), which are typical for plan-driven approaches as realized in traditional WFMS. By closely interweaving design and operation, the approaches referenced in the paper allow a more agile way of planning. Users are empowered to defer decisions regarding the exact control-flow to run-time, when more precise information becomes available. The support for partial workflows allowing users to defer decisions to run-time, the absence of over-specification, and more manoeuvering room for end users are considered as advantages commonly attributed to declarative processes. Authors argue that a declarative approach is particularly promising for dynamic and unpredictable processes. Despite these observations, declarative processes seem to be rudimentarily supported, whereas methods and tools for supporting imperative processes are rather advanced.

This paper proposes an approach towards better understandability and maintainability of declarative processes by adopting well-established techniques from the domain of software engineering. The ideas of test-driven development and automated acceptance testing are adopted to interweave process specification and process testing. During modelling, test cases balance the circumstantial/sequential information mismatch as well as improve understandability. During process execution, test cases help to document the reasons for process deviations and ensure that respective deviations can be easily considered during schema evolution. When the process model is adapted in the course of schema evolution, test cases ensure that the adaptations do not interfere with existing requirements. In addition, test cases help to identify which part of the model needs to be changed.

\section{OPERATION, EVALUATION, (RE)DESIGN}

The paper by Sturm, 'Supporting business process analysis via data warehousing', defines a method for constructing data warehouse schemata from business process specifications to facilitate the offline analysis of the business process execution. Sturm defines the relationships between the business process specification stage and the analysis/evaluation stages, with the aim to facilitate and to identify potential improvements by querying the business process performance. The evaluation stage may be realized online in terms of learning and changing the business process as it is executed, or it may take place offline via an online analytical process procedure for measuring the business process performance or via process mining approach. The author advocates that the online approach is suited to deal with exceptions and minor changes within the process execution, whereas the offline analysis of business processes should be applied for improvement of specific business processes. This work is about the way the source data is determined and collected for the purpose of applying various processmining techniques. Data warehousing is used during offline analysis for decision making by enabling users to view organizational data at various levels. Although various suites may be used for supporting the entire lifecycle of a business process, they do not provide guidelines as to what elements should be examined or analyzed in order to obtain better performance of the business processes and their organization.

This paper seeks to address this lack by examining the interrelationships between business process specification and business process analysis. The author suggests a method for transforming business process specifications into data warehouse schemata (i.e., snowflake schemata). This transformation focuses on the business process analysis as the most important information, thereby increasing the possibility of identifying potential improvements that should be introduced into the business process specifications.

The paper by Shahzad et al., 'Process warehouses in practice: a goal-driven method for business process analysis', also adresses an approach to analyzing business processes, which involves the use of process-oriented data warehouses to collect information about executed processes to enable their detailed analysis and thereby to provide bases for possible improvements. Authors draw our attention on the fact that any analysis aiming for process improvement requires detailed information about the running processes. Data warehouse technology together with analysis techniques have been widely 
used to enhance the analytical capabilities of enterprises. However, conventional data warehouses do not provide an adequate basis for a detailed analysis of business processes, because the warehouse data extracted from online transactional systems do not capture the information about ongoing process activities.

The process warehouse concept has been introduced as an alternative to the data warehouse - it is defined as a warehouse that stores data about executed business processes, such as the actors, the activities performed by the actors, the execution time of activities, the frequency of activities and the availability of resources, and thereby can be used as an adequate basis for the analysis and optimization of those processes. This study proposes a three-step method for the analysis of business processes and illustrates its application in a process from the health-care domain: (i) the process analysis requirements are defined on the business goal level; (ii) the produced goal structure is used to design a corresponding process warehouse; and (iii) the goal structure designed in the first step is used to choose the desired process analysis directions, by selecting a set of process-related goals and retrieving the information from the process warehouse that will enable the analysis of fulfilment of those goals.

The paper by De Nicola et al., 'Towards a method for business process and informal business rules compliance', addresses the transition from the evaluation phase to the (re)design phase, providing also a support to the (re)design phase itself. During the last two phases of the lifecycle, operation and evaluation, there are several elements that are collected and used in the next iteration of the cycle, aimed at (re)designing the new versions of the business processes. Several motivations may push an enterprise to intervene on its business processes. For instance, the change of laws and regulations that requires a different approach to the same activities; the change of company strategies, or undesired behaviours identified during the evaluation of the deployed business processes. Authors argue that some of the preceding cases can be expressed by using business rules and consider business rules as directives aimed at modifying the way an enterprise operates.

The paper addresses two key problems related to the use of business rules in business process reengineering. The first problem concerns the fact that general business rules are formulated by the top management and distributed by the organization unit of the enterprise, in natural language, often ambiguous and imprecise. The second problem concerns the identification of the business processes that are impacted by a business rule, locating the specific business process segments causing the violation of the business rule. Authors focus on a particular class of business rules, that is, dependency constraints (compliance rules) that impose the presence (or absence) of a certain activity when some other activity is performed, possibly imposing also ordering conditions, that is, precedence or subsequence of one another. They propose two solutions: one for natural language business rule disambiguation and one for automatic verification of business process/business rule compliance. They also propose a method and a tool to verify compliance of business rules against the existing business processes and identify the specific segments where the rule is violated.

\section{THE WHOLE LIFECYCLE}

The paper by Schmidt, 'Meta-service support for the business process lifecycle', addresses the service orientation in business process engineering. In fact, because of the increasing division of labour, offshoring, globalisation and so on, business processes more and more use services provided by other organizations instead of using on-premise functionality. Using technical innovations such as Web services and software as a service, it has become rather easy to use services provided by other organizations. The services used in business processes include not only services already known from SOA but also contain human based services, infrastructure service and so on. Often, higher services are provided by combining low-level services. However, the lifecycle of such service-based processes has to be augmented by functionalities to manage services. That means, in parallel to the lifecycle of the business processes containing operations such as the definition, deployment, operation and so on, equivalent operations have to take place for services. These service management operations, as part of a service life cycle, are applied to different services and therefore have to be defined separately. Furthermore, the service management operations have to comply with constraints originating from 
business, laws and so on. These constraints may not predefine the service management operations in detail; however, they define rules to be obeyed by all service management operations.

This paper introduces a new approach for managing services in the business process lifecycle called metaservices. The idea of metaservices is to capture the functionality to manage the services during the lifecycle in turn as service and thus to create a uniform interface both for services and their management. Metaservices encapsulate operations acting upon services as services themselves and thus to handle them equally to services. Furthermore, metaservices capture the structures and functionalities common to different of those operations. Redundant structures are avoided as with previous approaches. In this way, metaservices systematically and homogenously manage services during the business process lifecycle.

\title{
ACKNOWLEDGEMENTS
}

The BPMDS series has produced 11 workshops from 1998 to 2010. Nine of these workshops were held in conjunction with CAiSE conferences. From 2011, it will become a (two days) working conference attached to CAiSE. The topics addressed by the BPMDS series are focused on IT support for business processes. This is one of the keystones of information systems theory. The goals, format and history of BPMDS can be found on the website: http://bpmds.org/

We wish to thank the members of the Programme Committee, namely Ian Alexander, Renata Mendes de Araujo, Stewart Green, Paul Johannessen, Agnes Koshmider, Manfred Reichert, Pnina Soffer, and Lars Taxén for their help and constructive comments during the review process.

IAE de Paris, University Paris 1 Panthéon Sorbonne, 21, rue Broca, 75005 Paris, France

E-mail: nurcan@univ-paris1.fr

RAINER SCHMIDT Business Information Systems, Aalen University, Aalen, Germany

ILIA BIDER

IbisSoft, Stockholm, Sweden

GIL Regev

LAMS - Systemic Modeling Laboratory, EPFL and Itecor, Lausanne, Switzerland

\author{
AUTHORS' BIOGRAPHIES
}

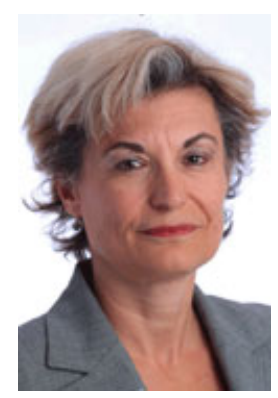

Selmin Nurcan is an Associate Professor at the University Paris 1 Panthéon Sorbonne and a Senior Researcher at the 'Centre de Recherche en Informatique'. She has a $\mathrm{PhD}$ and an Engineering degree in Computer Science. Her research activities include enterprise modelling, business process management, enterprise architecture and business/IS alignment, change modelling, business process (re) engineering and IS engineering and IS governance. She is author or co-author of more than 100 research papers, and is co-organizer of the Business Process Modeling, Development, and Support workshop series at CAiSE, the BPMS2 workshop series at BPM and the SoEA4EE workshop series at EDOC. She is acting as a programme committee member of a number of international and national conferences. She is serving on the editorial board of numerous international journals. She has been a guest editor of a number of journal special issues related to business process modelling, development and support, and to enterprise and information systems modelling. 


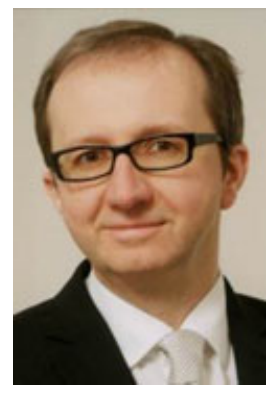

Rainer Schmidt is a Professor for business information systems at Aalen University. He has a $\mathrm{PhD}$ and an engineering degree in Computer Science. His current research areas are service science, business process management, social software and the integration of these themes. Rainer Schmidt is co-organizer of the BPMDS workshop series at Conference on Advanced Information Systems Engineering (CAiSE), the BPMS2 workshop series at BPM and the SoEA4EE workshop series at EDOC. Furthermore, he is member of the programme committee of several workshops and conferences. Rainer Schmidt is serving on the editorial boards of International Journal of Information Systems in the Service Sector and International Journal on Advances in Internet Technology. Rainer Schmidt applies his research in a number of projects and cooperations with industry. He has industrial experience as management consultant and researcher.

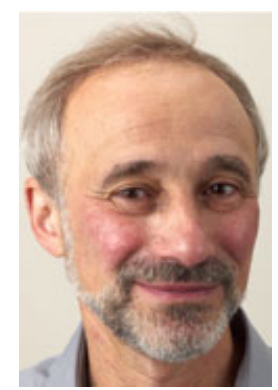

Ilia Bider is the director of R\&D and co-founder of IbisSoft. He is an active proponent of Douglas Engelbart's vision that the aim of a computer system is to enhance human intellect, rather than substitute it or turn humans into slaves. $\mathrm{He}$ has a combined experience of over 30 years of research and practical work in five countries. He is the inventor of the state-oriented business process modelling technique, and the author of many research papers, as well as articles for practitioners. He frequently holds tutorials at international conferences, and he sits on the editorial board of several academic journals.

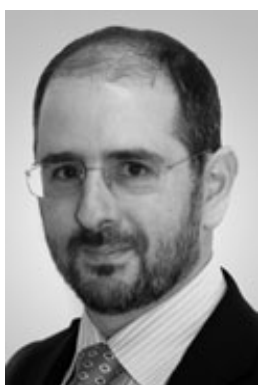

Gil Regev is a senior researcher at the School of Computer and Communication Sciences of the Ecole Polytechnique Fédérale de Lausanne (EPFL) and Knowledge Manager at Itecor, an IT consultancy firm based in Switzerland. Gil's research interests are in General Systems Thinking, which he applies to Enterprise Architecture, Requirements Engineering and Knowledge Management. Gil has a PhD in Enterprise Information Systems and an MSc in Computer Science from EPFL. Gil has over 20 years of experience in research and industry. 